\title{
Bernoulli polynomial and the numerical solution of high- order boundary value problems
}

\author{
Mohamed El-Gamel*, Waleed Adel, M. S. El-Azab \\ Department of Mathematics and Engineering Physics, Faculty of Engineering, Mansoura University, Egypt.
}

\begin{abstract}
In this work we present a fast and accurate numerical approach for the higher-order boundary value problems via Bernoulli collocation method. Properties of Bernoulli polynomial along with their operational matrices are presented which is used to reduce the problems to systems of either linear or nonlinear algebraic equations. Error analysis is included. Numerical examples illustrate the pertinent characteristic of the method and its applications to a wide variety of model problems. The results are compared to other methods.
\end{abstract}

Keywords: Bernoulli, collocation, higher-order, astrophysics, error analysis.

2010 MSC: 35G15, 74H15.

(C)2019 All rights reserved.

\section{Introduction}

A lot of real world applications including hydrodynamics, fluid dynamics, astronomy, astrophysics, beam and long wave theory are modeled by boundary value problems of high order. Due to their importance, these models have been investigated extensively for studying their behavior during the last decade. For example, when heating an infinite horizontal layer of fluid from below with the action of rotation, chandrasekhar [13] proves that instability occurs. When this happens, as overstability, it can be modeled by an eighth order problem. Also, these type of equations which occurs in torsional vibration of uniform beams have been investigated by Bishop et al. [10]. They are often used in the simulation of a lot of physical phenomena such as astrophysics or heat transfer problems which are known to be simulated by a sixth-order boundary value problem [11,33,48]. Also, in the modeling of viscoelastic or inelastic flows and deformation of beams, a fourth-order boundary value problem is used to model the effect of these flows or these deformations [15]. Further discussions of fourth, sixth and eighth-order problems are introduced in $[7,31]$.

The uniqueness and existence theorems of solutions of such problems are thoroughly discussed in [3] but no numerical methods are contained therein. However, there have been few numerical techniques

\footnotetext{
*Corresponding author

Email addresses: gamel_eg@yahoo.com (Mohamed El-Gamel), waleedade185@yahoo.com (Waleed Adel),

ms_elazab@hotmail.com (M. S. El-Azab)
}

doi: $10.22436 / \mathrm{mns} .04 .01 .05$

Received: 2018-11-28 Revised: 2019-03-03 Accepted: 2019-03-05 
which treats this types of problems. These methods include collocation methods [5], finite difference method [12, 14], sinc Galerkin [20], non-polynomial spline functions [46], octic spline functions [47], quintic spline [33], Adomian decomposition method [55, 56], reproducing kernel space method [4], differential quadrature method [35], variational iteration method [1, 45], differential transformation method [22], Homotopy perturbation method [25, 40,43], and the Galerkin residual technique with Bernstein and Legendre polynomials [30]. Recently, El-Gamel and Abdrabou [16] proposed a new approach for solving eight-order boundary value problems via sinc-Galerkin. Each of these methods has it's own advantages and drawbacks.

The aim of this work is to develop a solution of general form of higher-order boundary value problems

$$
\frac{d^{2 r} u(x)}{d x^{2 r}}+\sum_{m=0}^{2 r-1} \sigma_{m} \frac{d^{m} u(x)}{d x^{m}}=\psi(x, u), \quad 0 \leqslant x \leqslant 1, \quad r=2,3,4,
$$

subjected to the boundary conditions

$$
\left.\frac{d^{i} u(x)}{d x^{i}}\right|_{x=0}=\alpha_{i},\left.\quad \frac{d^{i} u(x)}{d x^{i}}\right|_{x=1}=\beta_{i}, \quad i=0,1,2, \ldots, r-1,
$$

where $\psi(x, u)$ and $u(x)$ are both continuous functions.

Over the past few years, Bernoulli polynomials gains an increasing importance in numerical analysis due to many reasons. The efficiency of these polynomials has been formally investigated in $[8,9,21$, $28,38,39,51-53]$ and can be used to treat other problems as in $[6,36,37]$. For more details about see $[17,24,42,51]$ and references therein.

To our first knowledge the results that are presented in this work are the first to be introduced for solving high-order boundary value problems using Bernoulli collocation method.

The outline of the paper is organized as follows. In Section 2, we present the basic concepts of these polynomials. In Section 3, Bernoulli collocation method is developed for both linear and nonlinear higherorder boundary value problems. Error analysis of the method is described in Section 4. In Section 5, a residual error correction is made. Some numerical examples are presented in Section 6. Finally, Section 7 is devoted for the conclusion of this study.

\section{Fundamental relations}

More detailed discussions about Bernoulli operational matrix can be found in [49, 50, 53].

\subsection{Bernoulli operational matrix of differentiation}

We will use Bernoulli approximation technique to approximate the solution of Eqs. (1.1)-(1.2) expressed in the following form

$$
u_{N}(x)=\sum_{n=0}^{N} c_{n} B_{n}(x)=\mathbf{B}(x) c,
$$

where $\left\{c_{n}\right\}_{n=0}^{N}$ are the unknown Bernoulli coefficients. We choose $N$ which is any chosen positive integer such that $N \geqslant 2 r$ and $B_{n}(x), n=0,1, \ldots, N$ are the Bernoulli polynomial of the first kind. Then, the Bernoulli coefficient vector $\mathbf{c}$ and the Bernoulli vector $\mathbf{B}(\mathrm{x})$ are given by

$$
\mathbf{c}^{\mathrm{t}}=\left[\mathrm{c}_{0}, \mathrm{c}_{1}, \ldots, \mathrm{c}_{\mathrm{N}}\right], \quad \mathbf{B}(\mathrm{x})=\left[\mathrm{B}_{0}(\mathrm{x}), \mathrm{B}_{1}(\mathrm{x}), \ldots, \mathrm{B}_{\mathrm{N}}(\mathrm{x})\right] .
$$

Then we have

$$
u_{N}^{(k)}=\mathbf{B}(x) \mathbf{M}^{k} \mathbf{c}, \quad k=1, \ldots, 2 r,
$$


where

$$
\mathbf{M}=\left[\begin{array}{ccccc}
0 & 1 & 0 & \ldots & 0 \\
0 & 0 & 2 & \ldots & 0 \\
\vdots & \vdots & \vdots & \ddots & \vdots \\
0 & 0 & 0 & \ldots & \mathrm{N} \\
0 & 0 & 0 & \ldots & 0
\end{array}\right]
$$

\section{Description of Bernoulli matrix collocation method}

We will illustrate our method based on Eq. (1.1) on two cases

\subsection{Linear high-order BVP}

Consider a linear, higher-order BVP of the form

$$
\frac{d^{2 r} u(x)}{d x^{2 r}}+\sum_{m=0}^{2 r-1} \sigma_{m} \frac{d^{m} u(x)}{d x^{m}}=f(x), \quad 0 \leqslant x \leqslant 1, \quad r=2,3,4 .
$$

The approximate solution for $u(x)$ is represented by the formula

$$
u(x) \approx u_{N}(x)=\sum_{n=0}^{N} c_{n} B_{n}(x)=\mathbf{B}(x) c,
$$

where the Bernoulli coefficient vector $\mathbf{c}$ and the Bernoulli vector $\mathbf{B}(x)$ are given

$$
\mathbf{c}^{\mathrm{t}}=\left[\mathrm{c}_{0}, \mathrm{c}_{1}, \ldots, \mathrm{c}_{\mathrm{N}}\right], \quad \mathbf{B}(\mathrm{x})=\left[\mathrm{B}_{0}(\mathrm{x}), \mathrm{B}_{1}(\mathrm{x}), \ldots, \mathrm{B}_{\mathrm{N}}(\mathrm{x})\right],
$$

then the $k^{\text {th }}$ derivative of $u_{N}(x)$ can be expressed in the matrix from by

$$
u_{N}^{(k)}(x)=\mathbf{B}^{(k)}(x) \mathbf{c}=\mathbf{B}(x) \mathbf{M}^{k} \mathbf{c}, \quad k=1,2, \ldots, 2 r .
$$

The nonhomogeneous term $f(x)$ of Eq. (3.1) can be expressed in terms of Bernoulli polynomials

$$
f(x) \approx \sum_{n=0}^{N} f_{n} B_{n}(x)=B(x) F,
$$

where $\mathbf{F}=\left[f_{0}, f_{1}, \ldots, f_{N}\right]^{t}$ and $f_{n}$ can be calculated from ([50])

$$
f_{n}=\frac{1}{n !} \int_{0}^{1} f^{(n)}(x) d x, \quad n=0,1, \ldots, N .
$$

We need the following theorem.

Theorem 3.1. If the assumed approximate solution of the boundary-value problem (3.1)-(1.2) is (2.1), then the discrete Bernoulli system for the determination of the unknown coefficients $\left\{\mathbf{c}_{n}\right\}_{n=0}^{N}$ is given by

$$
\sum_{n=0}^{N} c_{n} B_{n}^{(2 r)}\left(x_{k}\right)+\sum_{m=0}^{2 r-1} \sum_{n=0}^{N} c_{n} \sigma_{m} B_{n}^{(m)}\left(x_{k}\right)=\sum_{n=0}^{N} f_{n} B_{n}\left(x_{k}\right) .
$$

Proof. We replace each term in (3.1) with it's approximation defined in (2.1), (3.2) and use the collocation points $x=x_{k}$ defined by

$$
x_{k}=\frac{1}{N} k, \quad k=0,1, \ldots
$$


The matrix form of the Eq. (3.3) is

$$
\Phi \mathbf{c}=\mathbf{F}
$$

where

$$
\Phi=\left[\mathbf{M}^{2 r}+\sum_{m=0}^{2 r-1} \sigma_{m} \mathbf{M}^{m}\right] .
$$

The boundary conditions from Eq. (1.2) can be written in matrix form

$$
\mathbf{B}(0) \mathbf{M}^{i} \mathbf{c}=\left[\alpha_{i}\right], \quad \mathbf{B}(1) \mathbf{M}^{i} \mathbf{c}=\left[\beta_{i}\right], \quad i=0,1,2, \ldots, r-1 .
$$

These conditions can be simplified by writing (3.5) as in the form

$$
\bar{\Phi} \mathbf{c}=\overline{\mathbf{F}} .
$$

We acquire the new augmented matrix by replacing the $2 r$ rows of the matrix (3.4) with (3.6) and obtain the new augmented matrix

$$
[\bar{\Phi}, \overline{\mathrm{F}}]=\left[\begin{array}{cccccc}
\Phi_{00} & \Phi_{01} & \cdots & \Phi_{0 \mathrm{~N}} & \vdots & \mathrm{f}_{0} \\
\Phi_{10} & \Phi_{11} & \cdots & \Phi_{1 \mathrm{~N}} & \vdots & f_{1} \\
\Phi_{20} & \Phi_{21} & \cdots & \Phi_{2 \mathrm{~N}} & \vdots & f_{2} \\
\vdots & \vdots & \cdots & \vdots & \vdots & \vdots \\
\Phi_{\mathrm{N}-2 \mathrm{r}, 0} & \Phi_{\mathrm{N}-2 \mathrm{r}, 1} & \cdots & \Phi_{\mathrm{N}-2 \mathrm{r}, \mathrm{N}} & \vdots & \mathrm{f}_{\mathrm{N}-2 \mathrm{r}} \\
\mathrm{u}_{00} & \mathrm{u}_{01} & \cdots & \mathrm{u}_{0 \mathrm{~N}} & \vdots & \alpha_{0} \\
\vdots & \vdots & \cdots & \vdots & \vdots & \vdots \\
\mathrm{u}_{2 \mathrm{r}, 0} & \mathrm{u}_{2 \mathrm{r}, 1} & \cdots & \mathrm{u}_{2 \mathrm{r}, \mathrm{N}} & \vdots & \beta_{2 \mathrm{r}}
\end{array}\right]
$$

Now, we obtain a linear system of $N+1$ equations of the $N+1$ unknown coefficients. We can calculate those coefficients by solving this linear system. The system (3.7) may be easily solved by a different types of methods including the Q-R method that will be used in this paper.

\subsection{Nonlinear high-order BVP}

In this case we assign the source term $\psi(x, u)=f(x)-q(x)[u(x)]^{v}$ into Eq. (1.1) which will become

$$
\frac{d^{2 r} u(x)}{d x^{2 r}}+\sum_{m=0}^{2 r-1} \sigma_{m}(x) \frac{d^{m} u(x)}{d x^{m}}+q(x)[u(x)]^{v}=f(x), \quad 0 \leqslant x \leqslant 1, \quad r=2,3,4
$$

Then, by substituting $x=x_{k}$ collocation points mentioned in the previous section and the term $[u(x)]^{v}$ needed to be approximated first. For this, we need the following theorem.

Theorem 3.2 ([18]). The approximation of the function $\mathrm{u}^{v}\left(\mathrm{x}_{\mathrm{k}}\right), \mathrm{k}=0,1,2, \ldots$, can be represented according to

$$
\begin{aligned}
\left(\begin{array}{c}
u^{v}\left(x_{0}\right) \\
u^{v}\left(x_{1}\right) \\
\vdots \\
u^{v}\left(x_{N}\right)
\end{array}\right) & =\left(\begin{array}{ccccc}
u\left(x_{0}\right) & 0 & 0 & \ldots & 0 \\
0 & u\left(x_{1}\right) & 0 & \ldots & 0 \\
0 & 0 & u\left(x_{2}\right) & 0 & 0 \\
\vdots & \vdots & \ldots & \ddots & \vdots \\
0 & 0 & \ldots & 0 & u\left(x_{N}\right)
\end{array}\right)^{v-1}\left(\begin{array}{c}
u\left(x_{0}\right) \\
u\left(x_{1}\right) \\
\vdots \\
u\left(x_{N}\right)
\end{array}\right) \\
& =(\widetilde{\boldsymbol{u}})^{v-1} u=(\widetilde{B} \widetilde{\boldsymbol{C}})^{v-1}(B \boldsymbol{c})
\end{aligned}
$$


where

$$
\widetilde{\boldsymbol{B}}=\left(\begin{array}{ccccc}
\mathrm{B}\left(\mathrm{x}_{0}\right) & 0 & 0 & \ldots & 0 \\
0 & \mathrm{~B}\left(\mathrm{x}_{1}\right) & 0 & \ldots & 0 \\
0 & 0 & \mathrm{~B}\left(\mathrm{x}_{2}\right) & 0 & 0 \\
\vdots & \vdots & \ldots & \ddots & \vdots \\
0 & 0 & \ldots & 0 & \mathrm{~B}\left(\mathrm{x}_{\mathrm{N}}\right)
\end{array}\right), \quad \widetilde{\boldsymbol{C}}=\left(\begin{array}{ccccc}
\mathrm{c} & 0 & 0 & \ldots & 0 \\
0 & \mathrm{c} & 0 & \ldots & 0 \\
0 & 0 & \mathrm{c} & 0 & 0 \\
\vdots & \vdots & \ldots & \ddots & \vdots \\
0 & 0 & \ldots & 0 & \mathrm{c}
\end{array}\right) .
$$

By substituting the above theorem into Eq. (3.8), we reach the following theorem.

Theorem 3.3. If the assumed approximate solution of the problem (3.8) is (2.1), then the discrete Bernoulli system is

$$
\frac{d^{2 r} u\left(x_{k}\right)}{d x^{2 r}}+\sum_{m=0}^{2 r-1} \sigma_{m}\left(x_{k}\right) \frac{d^{m} u\left(x_{k}\right)}{d x^{m}}+q\left(x_{k}\right)\left[u\left(x_{k}\right)\right]^{v}=f\left(x_{k}\right), \quad 0 \leqslant x \leqslant 1, \quad r=2,3,4 .
$$

Proof. If we replace each term of (3.8) with its corresponding approximation given by (2.1) and (2.2) and substituting $x=x_{k}$ collocation points, the matrix form for the above system is

$$
\Theta \mathbf{c}=\mathbf{F},
$$

where

$$
\Theta=\left[\mathbf{M}^{2 r}+\sum_{m=0}^{2 r-1} \sigma_{m} \mathbf{M}^{m}\right] \mathbf{B}+\mathbf{Q}(\widetilde{\mathbf{B}} \widetilde{\mathbf{C}})^{\gamma-1} \mathbf{B}
$$

and

$$
\begin{aligned}
& \mathbf{F}=\left(\begin{array}{c}
f\left(x_{0}\right) \\
f\left(x_{1}\right) \\
f\left(x_{2}\right) \\
\vdots \\
f\left(x_{N}\right)
\end{array}\right), \quad \sigma_{\mathfrak{m}}=\left(\begin{array}{ccccc}
\sigma_{\mathfrak{m}}\left(x_{0}\right) & 0 & 0 & \ldots & 0 \\
0 & \sigma_{\mathfrak{m}}\left(x_{1}\right) & 0 & \cdots & 0 \\
0 & 0 & \sigma_{\mathfrak{m}}\left(x_{2}\right) & 0 & 0 \\
\vdots & \vdots & \cdots & \ddots & \vdots \\
0 & 0 & \cdots & 0 & \sigma_{\mathfrak{m}}\left(x_{N}\right)
\end{array}\right) . \\
& \mathbf{Q}=\left(\begin{array}{ccccc}
\mathrm{q}\left(\mathrm{x}_{0}\right) & 0 & 0 & \ldots & 0 \\
0 & \mathrm{q}\left(\mathrm{x}_{1}\right) & 0 & \ldots & 0 \\
0 & 0 & \mathrm{q}\left(\mathrm{x}_{2}\right) & 0 & 0 \\
\vdots & \vdots & \ldots & \ddots & \vdots \\
0 & 0 & \ldots & 0 & \mathrm{q}\left(\mathrm{x}_{\mathrm{N}}\right)
\end{array}\right), \quad \mathbf{B}=\left(\begin{array}{ccccc}
\mathrm{B}_{0}\left(\mathrm{x}_{0}\right) & \mathrm{B}_{1}\left(\mathrm{x}_{0}\right) & \mathrm{B}_{2}\left(\mathrm{x}_{0}\right) & \ldots & \mathrm{B}_{\mathrm{N}}\left(\mathrm{x}_{0}\right) \\
\mathrm{B}_{0}\left(\mathrm{x}_{1}\right) & \mathrm{B}_{1}\left(x_{1}\right) & \mathrm{B}_{2}\left(x_{1}\right) & \ldots & \mathrm{B}_{\mathrm{N}}\left(\mathrm{x}_{1}\right) \\
\mathrm{B}_{0}\left(\mathrm{x}_{2}\right) & \mathrm{B}_{1}\left(\mathrm{x}_{2}\right) & \mathrm{B}_{2}\left(\mathrm{x}_{2}\right) & \ldots & \mathrm{B}_{\mathrm{N}}\left(\mathrm{x}_{2}\right) \\
\vdots & \vdots & \vdots & \ddots & \vdots \\
\mathrm{B}_{0}\left(\mathrm{x}_{\mathrm{N}}\right) & \mathrm{B}_{1}\left(\mathrm{x}_{\mathrm{N}}\right) & \mathrm{B}_{2}\left(\mathrm{x}_{\mathrm{N}}\right) & \ldots & \mathrm{B}_{\mathrm{N}}\left(\mathrm{x}_{\mathrm{N}}\right)
\end{array}\right) .
\end{aligned}
$$

Then, by replacing the $2 r$ rows of the augmented matrix $[\Theta: \mathbf{F}]$ with the matrix representation by the boundary conditions from Eq. (3.5), we have a new augmented matrix in the form

$$
\tilde{\Theta} \mathbf{c}=\tilde{\mathbf{F}},
$$

which is a nonlinear system of $\mathrm{N}+1$ equations in $\mathrm{N}+1$ unknown coefficients $\mathbf{c}$. We can obtain these coefficients by solving the above nonlinear system using the algorithm mentioned in [18].

\section{Error analysis}

Theorem 4.1 ([49]). Assume that $\mathrm{f} \in \mathrm{L}^{2}[0,1]$ is enough smooth function and can be approximated by means of Bernoulli series $\sum_{n=0}^{N} f_{n} B_{n}(x)$, then the coefficients $f_{n}$ for $n=0,1, \ldots N$ associated with these approximations can be in the form

$$
f_{n}=\frac{1}{n !} \int_{0}^{1} f^{(n)}(x) d x .
$$


Proof. See [49].

Theorem 4.2 ([50]). Assume that one approximates the function $\mathrm{f}(\mathrm{x})$ which is smooth enough on $\mathrm{x} \in[0,1]$ by means of Bernoulli polynomials as presented in Theorem 4.1. Then the coefficients $\mathrm{f}_{\mathrm{n}}$ decays as follows

$$
\mathrm{f}_{\mathrm{n}} \leqslant \frac{\boldsymbol{F}_{\mathrm{n}}}{\mathrm{n} !}
$$

where $\boldsymbol{F}_{\mathrm{n}}=\sup _{\mathrm{x} \in[0,1]}\left|\mathrm{f}^{(\mathrm{n})}(\mathrm{x})\right|$.

Proof. See [50].

Theorem $4.3([2,32])$. suppose that $\mathrm{f}(\mathrm{x})$ belongs to the space $\mathrm{c}^{\mathrm{k}}[0,1]$ and is approximated by means of Bernoulli polynomials as stated in Theorem 4.2. Also, assume that $\mathrm{P}_{\mathrm{N}}[\mathrm{f}](\mathrm{x})$ is the polynomials of $\mathrm{f}(\mathrm{x})$ that are approximated in terms of Bernoulli polynomials and $\mathrm{R}_{\mathrm{N}}[\mathrm{f}](\mathrm{x})$ is the reminder term. Then,

$$
\begin{aligned}
f(x) & =P_{N}[f](x)+R_{N}[f](x), \quad x \in[0,1], \\
P_{N}[f](x) & =\int_{0}^{1} f(x) d x+\sum_{j=1}^{N} \frac{B_{j}(x)}{j !}\left(f^{(j-1)}(1)-f^{(j-1)}(0)\right), \\
R_{N}[f](x) & =-\frac{1}{N !} \int_{0}^{1} B_{N}^{*}(x-t) f^{(N)}(t) d t,
\end{aligned}
$$

where $\mathrm{B}_{\mathrm{N}}^{*}(\mathrm{x})=\mathrm{B}_{\mathrm{N}}(\mathrm{x}-[\mathrm{x}])$ and $[\mathrm{x}]$ denotes the largest integer not greater than $\mathrm{x}$.

Proof. See [2].

Theorem 4.4 ([52]). Suppose that $\mathrm{f}(\mathrm{x}) \in \mathrm{C}^{\infty}[0,1]$ (with uniformly bounded derivatives) and $\mathrm{P}_{\mathrm{N}}[\mathrm{f}](\mathrm{x})$ it's truncated series using Bernoulli polynomials. Then the error bound can be derived from the following relation

$$
\left\|R_{N}[f](x)\right\|_{\infty} \leqslant C \hat{F}(2 \pi)^{-N}, \quad x \in[0,1],
$$

where $\hat{F}$ denotes a bound for all the derivatives of the function $f(x)\left(i . e .,\left\|f^{(i)}(x)\right\|_{\infty} \leqslant \hat{F}\right.$ and for $\left.i=0,1,2, \ldots\right)$ and $\mathrm{C}$ is a positive constant.

Proof. See [52].

Theorem 4.5. Suppose that $u(x)$ be an enough smooth function and $u_{N}(x)$ be the truncated Bernoulli series of $\mathrm{u}(\mathrm{x})$. Then,

$$
\left\|\mathfrak{u}(x)-u_{N}(x)\right\|_{\infty} \leqslant G \hat{F}(2 \pi)^{-N}
$$

where $G$ is a positive constant.

Proof. An operator form can be written as

$$
L u=u^{(2 r)}=f(x)+g(x, u(x)),
$$

where the differential operator $L$ is given by

$$
L=\frac{d^{2 r}}{d x^{2 r}}
$$

The inverse operator $\mathrm{L}^{-1}$ is therefore considered a 2 -fold integral operator defined by

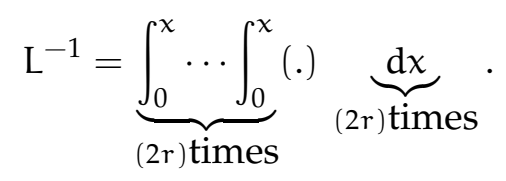


Operating with $\mathrm{L}^{-1}$ on (4.1) yields

$$
u(x)=\mathrm{L}^{-1} \mathrm{f}(\mathrm{x})+\mathrm{L}^{-1} \mathrm{~g}(\mathrm{x}, \mathrm{u})=\underbrace{\int_{0}^{x} \cdots \int_{0}^{x}}_{(2 \mathrm{r}) \text { times }} \mathrm{f}(\mathrm{x}) \underbrace{\mathrm{d} x}_{(2 \mathrm{r}) \text { times }}+\underbrace{\int_{0}^{x} \cdots \int_{0}^{x}}_{(2 \mathrm{r}) \text { times }} \mathrm{g}(x, \mathrm{u}) \underbrace{\mathrm{d} x}_{(2 r) \text { times }}=\mathrm{F}(\mathrm{x})+\mathrm{G}(x, \mathrm{u}(x)) .
$$

By approximating the functions $u(x)$ and $F(x)$ by the Bernoulli polynomials, therefore,

$$
u_{N}(x)=F_{N}(x)+G\left(x, u_{N}(x)\right),
$$

thus, by subtracting the last two equations we find

$$
\begin{aligned}
\left\|u(x)-u_{N}(x)\right\|_{\infty} & =\left\|F(x)-F_{N}(x)+G(x, u(x))-G\left(x, u_{N}(x)\right)\right\|_{\infty} \\
& =\left\|F(x)-F_{N}(x)\right\|_{\infty}+\left\|G(x, u(x))-G\left(x, u_{N}(x)\right)\right\|_{\infty}, \\
\left\|u(x)-u_{N}(x)\right\|_{\infty} & \leqslant\left\|F(x)-F_{N}(x)\right\|_{+} L_{G}\left\|u(x)-u_{N}(x)\right\|_{\infty},
\end{aligned}
$$

where $L_{G}$ is the Lipschitz constant for $G(x, u(x))$, then

$$
\left\|u(x)-u_{N}(x)\right\|_{\infty} \leqslant \frac{1}{1-L_{G}}\left\|F(x)-F_{N}(x)\right\|_{\infty} \leqslant \frac{1}{1-L_{G}}\left\|F(x)-P_{N}[f](x)\right\|_{\infty} .
$$

Using Theorem 4.4, yields

$$
\left\|u(x)-u_{N}(x)\right\|_{\infty} \leqslant \frac{C}{1-L_{G}}\left[\hat{F}(2 \pi)^{-N}\right] \leqslant G \hat{F}(2 \pi)^{-N},
$$

where $\mathbf{G}=\frac{C}{1-L_{G}}$. This theorem provides that the error of $u(x)$ which will give the desired solution when using enough values of $N$ will depend directly on the approximation of the function $f(x)$.

\section{Residual correction and error estimation}

In this section, we will give an error estimation for the Bernoulli collocation method and the residual correction of the Bernoulli approximate solution. For the purpose, we will define the residual function of the Bernoulli method as

$$
\mathfrak{R}_{\mathrm{N}}(x)=\mathbf{L}\left[\mathrm{u}_{\mathrm{N}}(x)\right]-f(x),
$$

where $u_{N}(x)$ which is the Bernoulli polynomial solution defined by the Eq. (2.1) is the approximate solution of the problem in Eq. (3.1) along with it's boundary conditions defined in Eq. (1.2). Hence $u_{N}(x)$ satisfies the problem

$$
\begin{array}{r}
\mathbf{L}\left[u_{N}(x)\right]=\frac{d^{2 r} u_{N}(x)}{d x^{2 r}}+\sum_{m=0}^{2 r-1} \sigma_{m} \frac{d^{m} u_{N}(x)}{d x^{m}}=f(x)+\Re_{N}(x), \\
\left.\frac{d^{i} u_{N}(x)}{d x^{i}}\right|_{x=0}=\alpha_{i},\left.\quad \frac{d^{i} u_{N}(x)}{d x^{i}}\right|_{x=1}=\beta_{i}, \quad i=0,1,2, \ldots, r-1 .
\end{array}
$$

Also, we will define

$$
\hat{e}_{N}(x)=u(x)-u_{N}(x),
$$

where $u(x)$ is the exact solution of Eq. (3.1) then we define the differential equation for the error as

$$
\mathbf{L}\left[\hat{e}_{N}(x)\right]=\mathbf{L}[\mathbf{u}(x)]-\mathbf{L}\left[\mathbf{u}_{N}(x)\right]=\mathfrak{R}_{N}(x)
$$

with the homogenous boundary conditions

$$
\hat{e}_{N}^{(i)}(0)=0, \quad \hat{e}_{N}^{(i)}(1)=0, \quad i=0,1,2, \ldots, r-1
$$


Then, the problem will be converted into the following error differential equation as

$$
\hat{e}_{N}^{(2 r)}(x)+\sum_{m=0}^{2 r-1} \sigma_{m} \hat{e}_{N}^{(m)}(x)=\Re_{N}(x),
$$

we note that from Eq. (5.2), the inhomogeneous boundary conditions from Eq. (5.1) have been deduced into homogenous boundary conditions. Solving the problem described by Eq. (5.3) as in Section 3.1, we get the approximation

$$
\hat{e}_{N}(x)=\sum_{n=0}^{N} \hat{c}_{n} B_{n}(x) .
$$

Here, $\hat{c}_{n}, n=0,1, \ldots, N$ are the determinate coefficients from the error problem and $\hat{e}_{N}(x)$ is the error function based on the residual function $\mathfrak{R}_{N}(x)$. Consequently, we obtain the corrected Bernoulli error function and the estimated error function $\hat{e}_{N}(x)$.

\section{Numerical examples}

In this section, we will apply our method to four test problems of both linear and nonlinear type which were reported in the literature $[19,23,26,27,29,34,41,44,54]$ to demonstrate the efficiency of our technique. Our main interest is to compare our results with other methods on the same problems in order to illustrate the performance of the proposed method. All computations were carried out using Matlab 2015 a on a personal computer. The maximum absolute error can be calculated according to the following equations

$$
\left\|\mathbf{e}_{\mathrm{N}}(x)\right\|=\max \left\|\mathfrak{u}(x)-u_{N}(x)\right\| .
$$

Example 6.1 ([34]). Consider the linear special fourth-order boundary value problem with the constant $c$ in the form

$$
\frac{d^{4} u}{d x^{4}}=(1+c) \frac{d^{2} u}{d x^{2}}-c u+\frac{1}{2} c x^{2}-1, \quad 0 \leqslant x \leqslant 1,
$$

subjected to the boundary conditions

$$
\left.u(x)\right|_{x=0}=\left.\frac{d u}{d x}\right|_{x=0}=1 ;\left.\quad u(x)\right|_{x=1}=\sinh (1)+\frac{3}{2},\left.\quad \frac{d u}{d x}\right|_{x=1}=\cosh (1)+1,
$$

whose exact solution is

$$
u(x)=1+\frac{1}{2} x^{2}+\sinh (x)
$$

We seek the approximate solution $u_{N}(x)$ by Bernoulli series for $N=6$ and $c=10$ is

$$
u_{N}(x)=c_{0} B_{0}(x)+c_{1} B_{1}(x)+\cdots+c_{5} B_{5}(x) .
$$

Here we have

$$
\mathbf{M}^{(2)}=\left[\begin{array}{ccccccc}
0 & 0 & 2 & 0 & 0 & 0 & 0 \\
0 & 0 & 0 & 6 & 0 & 0 & 0 \\
0 & 0 & 0 & 0 & 12 & 0 & 0 \\
0 & 0 & 0 & 0 & 0 & 20 & 0 \\
0 & 0 & 0 & 0 & 0 & 0 & 30 \\
0 & 0 & 0 & 0 & 0 & 0 & 0 \\
0 & 0 & 0 & 0 & 0 & 0 & 0
\end{array}\right], \quad \mathbf{M}^{(4)}=\left[\begin{array}{ccccccc}
0 & 0 & 0 & 0 & 24 & 0 & 0 \\
0 & 0 & 0 & 0 & 0 & 120 & 0 \\
0 & 0 & 0 & 0 & 0 & 0 & 360 \\
0 & 0 & 0 & 0 & 0 & 0 & 0 \\
0 & 0 & 0 & 0 & 0 & 0 & 0 \\
0 & 0 & 0 & 0 & 0 & 0 & 0 \\
0 & 0 & 0 & 0 & 0 & 0 & 0
\end{array}\right] .
$$


The augmented matrix according to Eq. (3.4) is

$$
[\Phi ; \mathbf{F}]=\left[\begin{array}{ccccccccc}
10 & 0 & -22 & 0 & 24 & 0 & 0 & ; & 0.66667 \\
0 & 10 & 0 & -66 & 0 & 120 & 0 & ; & 5 \\
0 & 0 & 10 & 0 & -132 & 0 & 360 & ; & 5 \\
0 & 0 & 0 & 10 & 0 & -220 & 0 & ; & 0 \\
0 & 0 & 0 & 0 & 10 & 0 & -330 & ; & 0 \\
0 & 0 & 0 & 0 & 0 & 10 & 0 & ; & 0 \\
0 & 0 & 0 & 0 & 0 & 0 & 10 & ; & 0
\end{array}\right] .
$$

From Eq. (3.5) the augmented matrix forms for the conditions are computed as

$$
\left[\Phi_{0} ; \alpha_{0}\right]=\left[\begin{array}{ccccccccc}
1 & \frac{-1}{2} & \frac{1}{6} & 0 & \frac{-1}{30} & 0 & \frac{1}{42} & ; & 1
\end{array}\right], \quad\left[\Phi_{1} ; \alpha_{1}\right]=\left[\begin{array}{ccccccccc}
0 & 1 & -1 & \frac{1}{2} & 0 & \frac{-1}{6} & 0 & ; & 1
\end{array}\right],
$$
and

$$
\left[\Phi_{2} ; \beta_{0}\right]=\left[\begin{array}{lllllllll}
1 & \frac{1}{2} & \frac{1}{6} & 0 & \frac{-1}{30} & 0 & \frac{1}{42} & ; & 2.6752
\end{array}\right], \quad\left[\Phi_{3} ; \beta_{1}\right]=\left[\begin{array}{lllllllll}
0 & 1 & 1 & \frac{1}{2} & 0 & \frac{-1}{6} & 0 & ; & 2.5341
\end{array}\right] .
$$

Thus, the augmented matrix given in Eq. (3.7) after replacing the last four rows and adding the boundary conditions is calculated as

$$
[\bar{\Phi} ; \overline{\mathbf{F}}]=\left[\begin{array}{ccccccccc}
10 & 0 & -22 & 0 & 24 & 0 & 0 & ; & 0.66667 \\
0 & 10 & 0 & -66 & 0 & 120 & 0 & ; & 5 \\
0 & 0 & 10 & 0 & -132 & 0 & 360 & ; & 5 \\
1 & \frac{-1}{2} & \frac{1}{6} & 0 & \frac{-1}{30} & 0 & \frac{1}{42} & ; & 1 \\
0 & 1 & -1 & \frac{1}{2} & 0 & \frac{-1}{6} & 0 & ; & 1 \\
1 & \frac{1}{2} & \frac{1}{6} & 0 & \frac{-1}{30} & 0 & \frac{1}{42} & ; & 2.6752 \\
0 & 1 & 1 & \frac{1}{2} & 0 & \frac{-1}{6} & 0 & ; & 2.5341
\end{array}\right] .
$$

The unknown Bernoulli coefficient is obtained as

$$
\mathbf{c}=\left[\begin{array}{l}
1.709746868 \\
1.675201194 \\
0.771540317 \\
0.195959718 \\
0.022628540 \\
0.009844412 \\
0.000754345
\end{array}\right]
$$

Thus, the approximate solution according to Eq. (2.1) is given by

$$
u_{6}(x)=0.000754 x^{6}+0.0075814 x^{5}-0.0000966 x^{4}+0.16710999 x^{3}+0.499852 x^{2}+x+1,
$$

which is close to the exact solution of the problem .

Table 1 exhibits the maximum absolute error for different values of $c$ and $N$. Table 2 shows the comparison of maximum absolute error for different values of $c$ produced by Haar-wavelet in [26], Differential transformation in [41], Reproductive Kernel method in [23], and Bernoulli method.

Also, Figure 1 demonstrates the approximate and exact solution at $\mathrm{N}=14$ and Figure 2 gives the error history at the same value of $\mathrm{N}$. From theses figures and tables one can conclude that our method obtained highly accurate solutions even when changing the value of the constant $\mathrm{c}$.

Table 1: Results for Example 6.1.

\begin{tabular}{|c|c|c|c|c|}
\hline $\mathrm{N}$ & $\mathrm{c}=10$ & $\mathrm{c}=100$ & $\mathrm{c}=1000$ & $\mathrm{c}=1000000$ \\
\hline 10 & $2.184029 \mathrm{E}-09$ & $1.199762 \mathrm{E}-09$ & $1.235723 \mathrm{E}-11$ & $1.446487 \mathrm{E}-11$ \\
\hline 12 & $5.401324 \mathrm{E}-11$ & $3.785194 \mathrm{E}-12$ & $7.971401 \mathrm{E}-14$ & $9.259260 \mathrm{E}-14$ \\
\hline 14 & $1.391554 \mathrm{E}-12$ & $4.884981 \mathrm{E}-14$ & $4.440892 \mathrm{E}-16$ & $6.661338 \mathrm{E}-16$ \\
\hline 16 & $3.508305 \mathrm{E}-14$ & $6.661338 \mathrm{E}-16$ & $4.440892 \mathrm{E}-16$ & $4.440892 \mathrm{E}-16$ \\
\hline 18 & $1.332268 \mathrm{E}-15$ & $6.661338 \mathrm{E}-16$ & $2.220446 \mathrm{E}-16$ & $4.440892 \mathrm{E}-16$ \\
\hline
\end{tabular}


Table 2: Comparison of maximum absolute error for Example 6.1.

\begin{tabular}{|c|c|c|c|c|}
\hline$\left\|\mathbf{e}_{\mathrm{N}}(\mathrm{x})\right\|$ & $\mathrm{c}=10$ & $\mathrm{c}=100$ & $\mathrm{c}=1000$ & $\mathrm{c}=1000000$ \\
\hline Bernoulli method, $\mathrm{N}=18$ & $1.33227 \mathrm{E}-15$ & $6.66134 \mathrm{E}-16$ & $2.22044 \mathrm{E}-16$ & $4.44089 \mathrm{E}-16$ \\
\hline Haar wavelet [26] & $1.7725 \mathrm{E}-07$ & - & - & $3.547 \mathrm{E}-09$ \\
\hline Reproductive Kernel [23] & $1.70 \mathrm{E}-09$ & - & - & $4.10 \mathrm{E}-10$ \\
\hline Differential transform[41] & $1.60 \mathrm{E}-08$ & $8.10 \mathrm{E}-10$ & $5.10 \mathrm{E}-10$ & $9.20 \mathrm{E}-03$ \\
\hline
\end{tabular}

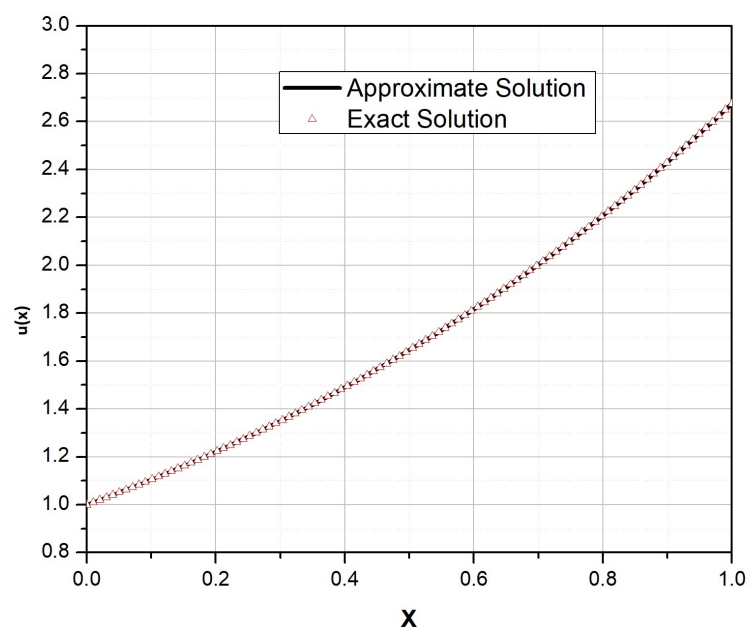

Figure 1: Approximate and exact solution at $\mathrm{c}=100$ and $\mathrm{N}=14$ for Example 6.1.

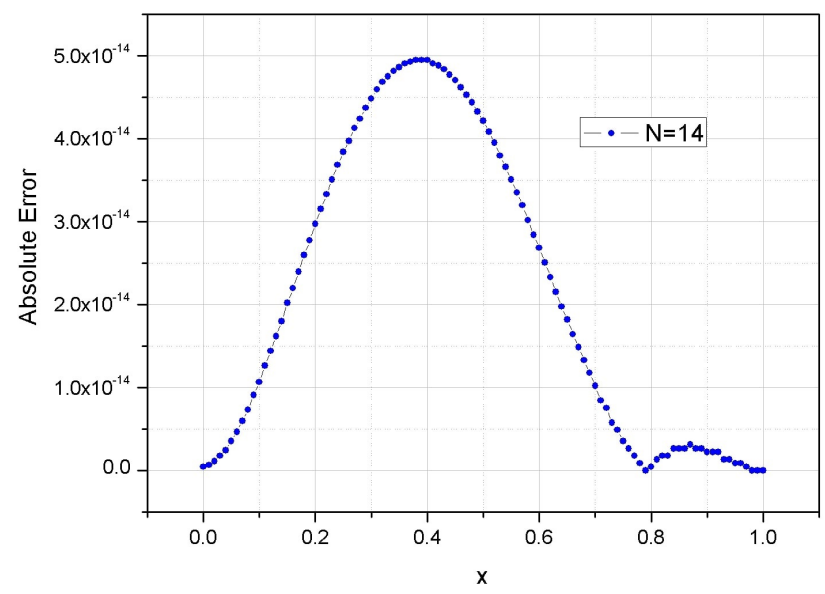

Figure 2: Error history at $\mathrm{c}=100$ and $\mathrm{N}=14$ for Example 6.1.

Example 6.2 ([44]). Now, consider the special form of linear sixth-order boundary value problem with the variable $\mathrm{c}$ according to Eq. (1.1) with $\mathrm{r}=3$ in the form

$$
\frac{d^{6} u}{d x^{6}}=(1+c) \frac{d^{4} u}{d x^{4}}-c \frac{d^{2} u}{d x^{2}}+c x, \quad 0 \leqslant x \leqslant 1,
$$

with the boundary conditions

$$
\left.u(x)\right|_{x=0}=\left.\frac{d u}{d x}\right|_{x=0}=1,\left.\quad \frac{d^{2} u}{d x^{2}}\right|_{x=0}=0,
$$




$$
\left.u(x)\right|_{x=1}=\sinh (1)+\frac{7}{6},\left.\quad \frac{d u}{d x}\right|_{x=1}=\cosh (1)+\frac{1}{2},\left.\quad \frac{d^{2} u}{d x^{2}}\right|_{x=1}=\sinh (1)+1,
$$

whose exact solution is

$$
u(x)=\sinh (x)+\frac{x^{3}}{6}+1
$$

Table 3 gives the maximum absolute error for different values of $\mathrm{c}$ and $\mathrm{N}$. Table 4 exhibits the comparison of maximum absolute error for different values of $c$ produced by Legendre-Galerkin [19], Haar Wavelet [27], Variational decomposition [44], Matlab dsolve toolbox [57], and Bernoulli method at $N=18$.

Table 3: Results for Example 6.2.

\begin{tabular}{|c|c|c|c|c|}
\hline $\mathrm{N}$ & $\mathrm{c}=10$ & $\mathrm{c}=100$ & $\mathrm{c}=1000$ & $\mathrm{c}=1000000$ \\
\hline 10 & $5.407641 \mathrm{E}-10$ & $6.815259 \mathrm{E}-11$ & $1.305534 \mathrm{E}-11$ & $1.511835 \mathrm{E}-11$ \\
\hline 12 & $1.461187 \mathrm{E}-11$ & $6.556977 \mathrm{E}-13$ & $8.459899 \mathrm{E}-14$ & $9.836576 \mathrm{E}-14$ \\
\hline 14 & $3.674838 \mathrm{E}-13$ & $6.883383 \mathrm{E}-15$ & $6.661338 \mathrm{E}-16$ & $6.661338 \mathrm{E}-16$ \\
\hline 16 & $9.547918 \mathrm{E}-15$ & $4.440892 \mathrm{E}-16$ & $4.440892 \mathrm{E}-16$ & $4.440892 \mathrm{E}-16$ \\
\hline 18 & $4.440892 \mathrm{E}-16$ & $2.220446 \mathrm{E}-16$ & $4.440892 \mathrm{E}-16$ & $4.440892 \mathrm{E}-16$ \\
\hline
\end{tabular}

Table 4: Comparison of maximum absolute error for Example 6.2.

\begin{tabular}{|c|c|c|c|c|}
\hline$\left\|\mathbf{e}_{\mathrm{N}}(\mathrm{x})\right\|$ & $\mathrm{c}=10$ & $\mathrm{c}=100$ & $\mathrm{c}=1000$ & $\mathrm{c}=1000000$ \\
\hline Bernoulli method, N=18 & $4.44089 \mathrm{E}-16$ & $2.22045 \mathrm{E}-16$ & $4.44089 \mathrm{E}-16$ & $4.44089 \mathrm{E}-16$ \\
\hline Variational decomposition [44] & $1.1 \mathrm{E}-05$ & $1.10 \mathrm{E}-03$ & $1.00 \mathrm{E}-01$ & $1.80 \mathrm{E}+02$ \\
\hline Legendre Galerkin [19] & $3.330 \mathrm{E}-15$ & $1.776 \mathrm{E}-15$ & $2.442 \mathrm{E}-15$ & $1.998 \mathrm{E}-15$ \\
\hline Haar Wavelet [27] & $1.6584 \mathrm{E}-9$ & $9.0835 \mathrm{E}-10$ & $2.3478 \mathrm{E}-10$ & $5.6208 \mathrm{E}-11$ \\
\hline Dsolve command [57] & $4.44089 \mathrm{E}-16$ & - & - & - \\
\hline
\end{tabular}

Example 6.3 ([29]). Now, we turn to another example of nonlinear eighth-order boundary value problem

$$
\frac{d^{8} u}{d x^{8}}=e^{-x} u^{2}, \quad 0 \leqslant x \leqslant 1,
$$

subjected to the boundary conditions

$$
\left.u(x)\right|_{x=0}=\left.\frac{d u}{d x}\right|_{x=0}=\left.\frac{d^{2} u}{d x^{2}}\right|_{x=0}=\left.\frac{d^{3} u}{d x^{3}}\right|_{x=0}=1,\left.\quad u(x)\right|_{x=1}=\left.\frac{d u}{d x}\right|_{x=1}=\left.\frac{d^{2} u}{d x^{2}}\right|_{x=1}=\left.\frac{d^{3} u}{d x^{3}}\right|_{x=1}=e,
$$

whose exact solution is

$$
u(x)=e^{x} .
$$

The absolute error for different values of $\mathrm{N}$ is presented in Table 5. Table 6 exhibits the comparison of maximum absolute error of Adomian method in [29] and Bernoulli method.

Table 5: Absolute maximum error for Example 6.3.

\begin{tabular}{|c|c|}
\hline $\mathrm{N}$ & $\left\|\mathbf{e}_{\mathrm{N}}(\mathrm{x})\right\|$ \\
\hline 10 & $1.75389 \mathrm{E}-11$ \\
\hline 12 & $5.24025 \mathrm{E}-14$ \\
\hline 14 & $8.88178 \mathrm{E}-16$ \\
\hline 16 & $8.88178 \mathrm{E}-16$ \\
\hline
\end{tabular}

Table 6: Comparison of absolute error for Example 6.3.

\begin{tabular}{|l|l|}
\hline Bernoulli method, $\mathrm{N}=16$ & Adomian Decomposition [29]
\end{tabular}

\begin{tabular}{l|l}
$8.88178 \mathrm{E}-16$ & $2.540 \mathrm{E}-05$
\end{tabular}


Example 6.4 ([54]). Another example is also a nonlinear eighth-order boundary value problem

$$
\frac{d^{8} u}{d x^{8}}+\exp (-x) u^{2}=\exp (-x)+\exp (-3 x), \quad 0 \leqslant x \leqslant 1,
$$

subjected to the boundary conditions

$$
\begin{aligned}
& \left.u(x)\right|_{x=0}=1,\left.\quad u(x)\right|_{x=1}=\exp (-1),\left.\quad \frac{d u}{d x}\right|_{x=0}=-1,\left.\quad \frac{d u}{d x}\right|_{x=1}=-\exp (-1), \\
& \left.\frac{d^{2} u}{d x^{2}}\right|_{x=0}=1,\left.\quad \frac{d^{2} u}{d x^{2}}\right|_{x=1}=\exp (-1),\left.\quad \frac{d^{3} u}{d x^{3}}\right|_{x=0}=-1,\left.\quad \frac{d^{3} u}{d x^{3}}\right|_{x=0}=-\exp (-1),
\end{aligned}
$$

with the exact solution

$$
u(x)=e^{-x}
$$

The absolute error for different values of $\mathrm{N}$ is presented in Table 7 and Table 8 exhibits the comparison of maximum absolute error along with Galerkin method in [54] and Bernoulli method. Also, Figure 3 shows the approximate solution along with the exact solution at $\mathrm{N}=14$.

Table 7: Absolute maximum error for Example 6.4.

\begin{tabular}{|c|c|}
\hline $\mathrm{N}$ & $\left\|\mathbf{e}_{\mathrm{N}}(\mathrm{x})\right\|$ \\
\hline 10 & $6.45195 \mathrm{E}-12$ \\
\hline 12 & $1.93179 \mathrm{E}-14$ \\
\hline 14 & $2.22045 \mathrm{E}-16$ \\
\hline 16 & $6.66134 \mathrm{E}-16$ \\
\hline
\end{tabular}

Table 8: Comparison of absolute error for Example 6.4.

\begin{tabular}{|c|c|}
\hline Bernoulli method, $\mathrm{N}=16$ & Galerkin method [54] \\
\hline 6.66134E-16 & $3.6418 \mathrm{E}-05$ \\
\hline
\end{tabular}

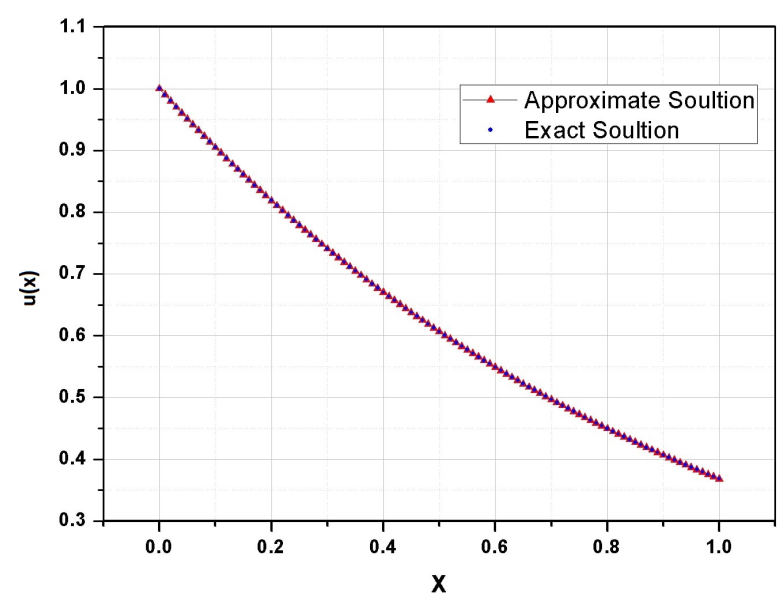

Figure 3: Approximate and exact solutions for $\mathrm{N}=14$ of Example 6.4. 


\section{Conclusion}

This paper discusses how Bernoulli matrix collocation method can be applied for obtaining solutions of linear and nonlinear higher-order boundary value problems. Our method is tested on examples along with a comparison with other methods including Matlab command Dsolve [57]. It is shown that Bernoulli collocation method yields good results.

Bernoulli collocation is a simple, straightforward and accurate method that can be used for solving such wide class of similar linear and nonlinear boundary value problems.

\section{Acknowledgment}

The authors would like to thank the referees for their valuable suggestions and comments.

\section{References}

[1] S. Abbasbandy, A. Shirzadi, The variational iteration method for a class of eighth-order boundary-value differential equations, Z. Naturforsch. A, 63 (2008), 745-751. 1

[2] M. Abramowitz, I. A. Stegun, Handbook of mathematical functions with formulas, Graphs, and Mathematical Tables, John Wiley \& Sons, New York, (1972). 4.3, 4

[3] R. P. Agarwal, Boundary Value Problems for Higher Order Differential Equations, World Scientific Publishing Co., Teaneck, (1986). 1

[4] G. Akram, H. U. Rehman, Numerical solution of eighth order boundary value problems in reproducing kernel space, Numer. Algorithms, 62 (2013), 527-540. 1

[5] U. M. Asher, R. M. M. Mattheij, R. D. Russel, Numerical solution of boundary value problems for ordinary differential equations, Siam, Philadelphia, (1995). 1

[6] E. Babolian, Z. Masouri, Direct method to solve Volterra integral equation of the first kind using operational matrix with block-pulse functions, J. Comput. Appl. Math., 220 (2008), 51-57. 1

[7] P. Baldwin, Asymptotic estimates of the eigenvalues of a sixth-order boundary value problem obtained by using global phase-integral methods, Philos. Trans. Roy. Soc. London Ser. A, 322 (1987), 281-305. 1

[8] A. H. Bhrawy, E. Tohidi, F. Soleymani, A new Bernoulli matrix method for solving high-order linear and nonlinear Fredholm integro-differential equations with piecewise intervals, Appl. Math. Comput., 219 (2012), 482-497. 1

[9] K. E. Biçer, S. Yalçinbaş, Numerical solution of telegraph equation using Bernoulli collocation method, Proc. Natl. Acad. Sci. India. Sect. A: Phys. Sci., 2018 (2018), 1-7. 1

[10] R. E. D. Bishop, S. M. Cannon, S. Miao, On coupled bending and torsional vibration of uniform beams, J. Sound Vib., 131 (1989), 457-464. 1

[11] A. Boutayeb, E. H. Twizell, Numerical methods for the solution of special sixth-order boundary value problems, Int. J. Comput. Math., 45 (1992), 207-233. 1

[12] A. Boutayeb, E. H. Twizell, Finite difference methods for the solution of eighth-order boundary value problems, Int. J. Comput. Math., 48 (1993), 63-75. 1

[13] S. Chandrasekhar, Hydrodynamic and Hydromagnetic Stability, Clarendon Press, Oxford, (1961). 1

[14] M. M. Chawla, C. P. Katti, Finite difference methods for two-point boundary value problems involving higher order differential equations, BIT Numer. Math., 19 (1979), 27-33. 1

[15] A. R. Davies, A. Karageoghis, T. N. Phillips, Spectral Galerkin methods for the primary two-point boundary value problem in modelling viscoelastic flows, Int. J. Numer. Math. Eng., 26 (1988), 647-652. 1

[16] M. El-Gamel, A. Abdrabou, Sinc-Galerkin solution to eighth-order boundary value problems, SeMA J., 76 (2019), 249 270. 1

[17] M. El-Gamel, W. Adel, Numerical investigation of the solution of higher-order boundary value problems via Euler matrix method, SeMA J., 75 (2018), 349-364. 1

[18] M. El-Gamel, W. Adel, M. S. El-Azab, Collocation method based on Bernoulli polynomial and shifted Chebychev for solving the Bratu equation, J. Appl. Computat. Math., 7 (2018), 7 pages. 3.2, 3.2

[19] M. El-Gamel, M. S. El-Azab, M. Fathy, The numerical solution of sixth order boundary value problems by the Legendre Galerkin method, Acta Univ. Apulensis Math. Inform., 40 (2014), 145-165. 6, 6.2, 4

[20] M. El-Gamel, A. I. Zayed, Sinc-Galerkin method for solving non-linear boundary value problems, Comput. Math. Appl., 48 (2004), 1285-1298. 1

[21] K. Erdem, S. Yalçinbaş, M. Sezer, A Bernoulli polynomial approach with residual correction for solving mixed linear Fredholm integro-differential-difference equations, J. Difference Equ. Appl., 19 (2013), 1619-1631. 1

[22] V. S. Ertürk, S. Momani, Comparing numerical methods for solving fourth-order boundary value problems, Appl. Math. Comput., 188 (2007), 1963-1968. 1 
[23] F. Z. Geng, A new reproducing kernel Hilbert space method for solving nonlinear fourth-order boundary value problems, Appl. Math. Comput., 213 (2009), 163-169. 6, 6.1, 2

[24] A. Golbabai, S. P. A. Beik, An efficient method based on operational matrices of Bernoulli polynomials for solving matrix differential equations, Comput. Appl. Math., 34 (2015), 159-175. 1

[25] A. Golbabai, M. Javidi, Application of homotopy perturbation method for solving eighth-order boundary value problems, Appl. Math. Comput., 191 (2007), 334-346. 1

[26] F. Haq, A. Ali, Numerical solution of fourth-order boundary value problems using Haar Wavelets, Appl. Math. Sci. (Ruse), 5 (2011), 3131-3146. 6, 6.1, 2

[27] F. Haq, A. Ali, I. Hussain, Solution of sixth-order boundary-value problems by collocation method using Haar wavelets, Int. J. Phys. Sci., 7 (2012), 5729-5735. 6, 6.2, 4

[28] E. Hesameddini, M. Riahi, Bernoulli Galerkin matrix method and its convergence analysis for solving system of volterrafredholm integro-differential Equations, Iran. J. Sci. Technol. Trans. Sci., 43 (2019), 1203-1214. 1

[29] M. Inc, D. J. Evans An efficient approach to approximate solutions of eighth-order boundary value problems, Int. J. Comput. Math., 81 (2004), 685-692. 6, 6.3, 6

[30] M. S. Islam, M. B. Hossain, Numerical solutions of eighth-order bop by the Galerkin residual technique with Bernstein and Legendre polynomials, Appl. Math. Comput., 261 (2015), 48-59. 1

[31] M. S. Islam, I. A. Tirmizi, F. Haq, M. A. Khan, Non-polynomial splines approach to the solution of sixth-order boundaryvalue problems, Appl. Math. Comput., 195 (2008), 270-284. 1

[32] V. I. Krylov, Approximate calculations of integrals, The Macmillan Co., New York-London, (1962). 4.3

[33] F.-G. Lang, X.-P. Xu, An effective method for numerical solution and numerical derivatives for sixth order two-Point boundary value problems, Comput. Math. Math. Phys., 55 (2015), 811-822. 1

[34] S. X. Liang, D. J. Jeffrey, An efficient analytical approach for solving fourth-order boundary value problems, Comput. Phys. Comm., 180 (2009), 2034-2040. 6, 6.1

[35] G. R. Liu, T. Y. Wu, Differential quadrature solutions of eighth-order boundary value differential equations, J. Comput. Appl. Math., 145 (2002), 223-235. 1

[36] K. Maleknejad, R. Mollapourasl, M. Alizadeh, Numerical solution of the Volterra type integral equation of the first kind with wavelet basis, Appl. Math. Comput., 194 (2007), 400-405. 1

[37] K. Maleknejad, B. Rahimi, Modification of block pulse functions and their application to solve numerically Volterra integral equation of the first kind, Commun. Nonlinear Sci. Numer. Simul., 16 (2011), 2469-2477. 1

[38] M. Matinfar, H. A. Lashaki, M. Akbari, Numerical approximation based on the Bernoulli polynomials for solving volterra integro-differential equations of high order, Math. Rese., 3 (2017), 19-32. 1

[39] F. Mirzaee, S. Bimesl, An efficient numerical approach for solving systems of high-order linear Volterra integral equations, Scientia Iranica, 21 (2014), 2250-2263. 1

[40] S. T. Mohyud-Din, M. A. Noor, Homotopy perturbation method for solving fourth-order boundary value problems, Math. Probl. Eng., 2007 (2007), 15 pages. 1

[41] S. Momani, M. A. Noor, Numerical comparison of methods for solving a special fourth-order boundary value problem, Appl. Math. Comput., 191 (2007), 218-224. 6, 6.1, 2

[42] A. Napoli, Solutions of linear second order initial value problems by using Bernoulli polynomials, Appl. Numer. Math., 99 (2016), 109-120. 1

[43] M. A. Noor, S. T. Mohyud-Din, Homotopy perturbation method for solving sixth-order boundary value problems, Comput. Math. Appl., 55 (2008), 2953-2972. 1

[44] M. A. Noor, K. I. Noor, S. T. Mohyud-Din, Variational iteration method for solving sixth-order boundary value problems, Commun. Nonlinear Sci. Numer. Simul., 14 (2009), 2571-2580. 6, 6.2, 4

[45] M. G. Porshokouhi, B. Ghanbari, M. Gholami, M. Rashidi, Numerical solution of eighth-order boundary value problems with variational iteration method, Gen. Math. Notes, 2 (2011), 128-133. 1

[46] S. S. Siddiqi, G. Akram, Solution of eighth-order boundary value problems using the non-polynomial spline technique, Int. J. Comput. Math., 84 (2007), 347-368. 1

[47] S. S. Siddiqi, E. H. Twizell, Spline solutions of linear eighth-order boundary value problems, Comput. Methods Appl. Mech. Eng., 131 (1996), 309-325. 1

[48] S. S. Siddiqi, E. H. Twizell, Spline solutions of linear sixth-order boundary value problems, Int. J. Comput. Math., 60 (1996), 295-304. 1

[49] E. Tohidi, A. H. Bhrawy, K. Erfani, A collocation method based on Bernoulli operational matrix for numerical solution of generalized pantograph equation, Appl. Math. Model., 37 (2013), 4283-4294. 2, 4.1, 4

[50] E. Tohidi, K. Erfani, M. Gachpazan, S. Shateyi, A new Tau method for solving nonlinear Lane-Emden type equations via Bernoulli operational matrix of differentiation, J. Appl. Math., 2013 (2013), 9 pages. 2, 3.1, 4.2, 4

[51] E. Tohidi, A. Kiliman, A Collocation method Based on the Bernoulli operational matrix for solving nonlinear BVPs which arise from the problems in calculus of variation, Math. Probl. Eng., 2013 (2013), 9 pages. 1

[52] F. Toutounian, E. Tohidi, A new Bernoulli matrix method for solving second order linear partial differential equations with the convergence analysis, Appl. Math. Comput., 223 (2013), 298-310. 4.4, 4

[53] F. Toutounian, E. Tohidi, S. Shateyi, A collocation method based on Bernoulli operational matrix for solving high-order linear complex differential equations in a rectangular domain, Abstr. Appl. Anal., 2013 (2013), 12 pages. 1, 2 
[54] K. N. S. K. Viswanadham, S. Ballem, Numerical solution of eighth-order boundary value problems by Galerkin method with quintic B-splines, Int. J. Comput. Appl., 89 (2014), 8880-8887. 6, 6.4, 8

[55] A.-M. Wazwaz, The numerical solutions of special eighth-order boundary value problems by the modified decomposition method, Neural Parallel Sci. Comput., 8 (2000), 133-146. 1

[56] A.-M. Wazwaz, The numerical solution of special fourth-order boundary value problems by the modified decomposition method, Int. J. Comput. Math., 79 (2002), 345-356. 1

[57] www.mathworks.com/help/symbolic/dsolve.html. 6.2, 4, 7 